\title{
Correlation between Voids Ratio and Characteristic Responses to Ultrasonic Pulses Through Sandy Soils
}

\author{
Fernanda V. Gonçalves ${ }^{1}$, Duilio N. Ferronatto Leite $^{1}$ \& José A. de Castro ${ }^{1}$ \\ ${ }^{1}$ Federal Fluminense University. Volta Redonda School of Metallurgical Engineering, Brazil \\ Correspondence: Fernanda V. Gonçalves, Federal Fluminense University. Volta Redonda School of Metallurgical \\ Engineering. Av. dos Trabalhadores 420 - Vila Sta. Cecília 27255-125 - Volta Redonda - RJ - Brazil. E-mail: \\ fevescovi@hotmail.com
}

Received: June 5, 2020

Accepted: July 2, 2020

Online Published: September 30, 2020

doi:10.5539/jmsr.v9n3p13

URL: https://doi.org/10.5539/jmsr.v9n3p13

\begin{abstract}
The microporous structure of granular soils that provides important information such as shear strength, compressibility, and hydraulic conductivity, is directly influenced by the void ratio. Therefore, a quick identification of the void ratio, by a non-destructively way and in situ becomes an interesting practice. Ultrasound has been successfully used for ceramic materials, wood, concrete and rocks. When dealing with soils, great efforts are made to understand its behavior and characteristics through wave propagation velocity. However, does still have a lack of discussions about the ultrasonic wave properties. In this paper, a study analysing 156 saturated samples of sands by ultrasound for 3 median particle size, with void ratio ranging from 0.5 to 1.1 . The ultrasonic wave measurement was performed using the transmit and receiveand technique, with $50 \mathrm{kHz}$ transducers. The study demonstrates a possibility of identifying transition zones between sample's materials composition. Considering the same dominant medium, a correlation was observed among the void ratio and the maximum amplitude, the damping coefficient, dominant frequency and ultrasonic pulse speed. There is also the identification of characteristic frequencies for these media. Therefore, the novelties of the present study are mainly the development of a feasible technique to investigate void ratio of granular saturated soils using direct measurements of the ultrasonic wave characteristics.
\end{abstract}

Keywords: Sandy Soils, Ultrasonic Characteristics, Amplitude, Damping Coefficient

\section{Introduction}

Sandy soils are important engineering materials used, for example, fill slopes, levees and rockfills dams (Zhang \& $\mathrm{Li}, 2010)$. They are also the main materials found in natural colluvial slopes. Therefore, investigation of the microporous structure can provide important information, such as shear strength, compressibility and hydraulic conductivity.

The void ratio (e) and porosity are characterstics the directly influence in microporous structure of granular soils and are dependent on the distribution of grains, as widely cited in the literature. In addition, the properties such as maximum and minimum voids, shear strength and compressibility parameters are also influenced by the shape of the particles (Das \& Shoban, 2014). Therefore, a quick identification of the void ratio, by a non-destructively way and in situ becomes an interesting practice.

Among waves propagation techniques, the ultrasound has been used successfully for studies in concrete (Wiggenhauser et al., 2017), determination of cracks in ceramic materials (Canivell, Martin-del-Rio, Alejandre, García-Heras \& Jimenez-Aguilar, 2018), wood and mortar (Pu, Hong, Liu, Xu \& Shan, 2019) and the mechanics of rocky materials (Nourani, Moghadder \& Safari, 2017).

Some applications of ultrasound techiniques on soil can be mention by Prasad and Meissner (1992) where they presented the possibility of using ultrasound in granular soils. Makse, Gland, Johnson and Schwartz (2004) show the use of ultrasonic pulse velocity to analyze the properties of soil and rocky materials. Velocity variations on ultrasonic pulse were observed regarding to volume, shear and contact between the particles. Zimmer (2004) measured the velocity for dry and saturated sands, with porosity ranging from 0.24 to 0.44 , verifying a small difference for dry samples at a given pressure. On the other hand, for saturated samples, the P-waves velocity show a significant growth with a decrease of porosity. 
Dutta, Otsubo, Kuwano and O' Sullivan (2019) showed that the P-waves and S-waves are independent of median particle size $\left(\mathrm{D}_{50}\right)$ of granular material. Xu, Cheng and Ling (2013) present values that are linearly correlating Swave velocity and void ratio for different stresses. Choobbasti, Samakoosh and Kutanaei (2019) present equations that relate unconfined compressive strength and ultrasonic pulse velocity for reinforced clayey soils.

Concerning to physical properties of ultrasonic waves Knuth, Tobin and Marone (2013) show a dependent relationship between characteristics and mechanical energy travels through granular soils. Gheibe and Hedayat (2018) show that the amplitude provides valuable informations regarding to soil compressibility. They conclude that P-waves travel more frequently through fine granular soil and also Pyrak-Nolt and Nolte (1992) show a correlation between frequency and fracture in rocks.

As mentioned by Gheibe and Hedayat (2018) there are great efforts to understand the effect on the deformation of soil particles through propagation velocity of ultrasonic waves, however, there are poorly discussions about deformation mechanisms in granular materials represented by ultrasonic wave properties. Therefore, the novelties of the present study are mainly the development of an accurate and feasible technique to investigate void ratio of granular saturated soils using direct measurements of the ultrasonic wave characteristics.

\section{Method}

\subsection{Material Acquisition and Characterization}

This paper used sand extracted from Volta Redonda city located in Rio de Janeiro state, Brazil. Three different types of sand were used. Type one: sand with grain size ranging from $0.59 \mathrm{~mm}$ to $0.074 \mathrm{~mm}$ and median particle size $\left(\mathrm{D}_{50}\right)$ equal to $0.4 \mathrm{~mm}$. Type two: sand with grains ranging from $2.0 \mathrm{~mm}$ to $0.6 \mathrm{~mm}$ and $\mathrm{D}_{50}=1 \mathrm{~mm}$ and, type three; sand with grains ranging from $1.18 \mathrm{~mm}$ and $0.6 \mathrm{~mm}$ and $\mathrm{D}_{50}=1.15 \mathrm{~mm}$. The samples were composed by saturated sand molded for ultrasound tests using aluminum capsules with $60 \mathrm{~mm}$ in length and $50 \mathrm{~mm}$ in diameter. The variation on void ratio was obtained by carefully varying the intensity of vibration of aluminum capsule when adding water and sand.

\subsection{Ultrasonic Measurements and Data Acquisition Procedure}

The technique of Transmit and Receiveand were applied employing two $50 \mathrm{kHz}$ transducers and the Panametrics ${ }^{\circledR}$ equipment model 5058PR High-voltage Ultrasonic Pulser-Receiver. According to Olympus (2016), this equipment was specially designed for tests and measurements in attenuating material media, being recommended for analysis of concrete, stone, soil and sands. At the end, acquiring 156 waveforms by using 52 samples.

To manage and treat the generated waveforms data analysis, a computational code based on logarithmic decrement definition was developed in Matlab ${ }^{\circledR}$ language. Its main purpose was to manually select two points, the frist largest peak and a second $\mathrm{n}$ periods away peak of waveform, helping to calculate the logarithmic decrement. According to Ferronatto (2016), the process can be optimized despite being done manually.

The logarithmic decrement calculation (Equation 1), to obtain the damping coefficient (Equation 2) was performed and compared with the void ratio.

$$
\Psi=\ln U_{1} / \ln U_{2}
$$

$\Psi \Psi$ is the logarithmic decrement; $U_{I} \mathrm{U}_{1}$ is the amplitude of the first largest peak; and $U_{2} \mathrm{U}_{2}$ is the amplitude of the nth peak after the loss of amplitude of the captured waveform.

$$
\zeta=\Psi / \sqrt{ } 4 \pi 2 n 2+(\Psi 2)
$$

$\zeta$ is the damping factor or coefficient for under-damped systems.

$n$ is the number of periods of the second acquired point, far from the amplitude of the first largest peak.

Through the computational code, it was possible to carry out the analysis of the waveform in the frequency domain based on fast Fourier transform (FFT), making it possible to convert the waveform in the time domain into a spectrum in the frequency domain. It was also possible to calculate the wave propagation time for the sample using the Transmit and Receive technique.

Equation 3 was used to calculate ultrasonic pulse velocity.

$$
U P V=\Delta S / \Delta t
$$

$\Delta t \Delta \mathrm{t}$ represents wave propagation time for the sample and $\Delta S \Delta$ Sis sample size.

The software Statistica8.0 produced by the company Statsoft was used to interpret the results and plot the diagrams. The analysis of the interaction between the different variables was performed using the software Statistica8.0 to 
treat and correlate the data with the parameters related with the wave characteristics and the void ratio of the granular material. The data analysis and statitiscal correlations are used to obtain the combination of values to construct the surface response model.

\section{Results and Discussion}

\subsection{Damping Coefficient}

Figure 1 shows waveforms of saturated sand samples of $\mathrm{D}_{50}=0.40 \mathrm{~mm}$ for differents voids ratio and also a fitted damping curve. A similar behavior was noted for other sands of $\mathrm{D}_{50}=1.00 \mathrm{~mm}$ and $\mathrm{D}_{50}=1.15 \mathrm{~mm}$. For all analyzed samples, there were a variation on waveform. Figure 1 (a) with $\mathrm{e}=0.51$, has significant reduction on amplitude and propagation wave time, when compared to other samples. Samples with void ratio 0.74, Figure 1(c), observed an increasing on wave propagation time and decreasing on amplitude. Figure 1(d) and (e) shows greatest spread, significant increasing on wave propagation time and amplitude. Then increasing the amount of water makes the medium grains of sand more distant and leads to an interpretation of discontinuity in the material, characterizing a greater wave scattering. Then increasing the amount of water means to spread the grains of sand, putting them away from each other and it leads to an interpretation of discontinuity in the material, characterizing a greater wave scattering.

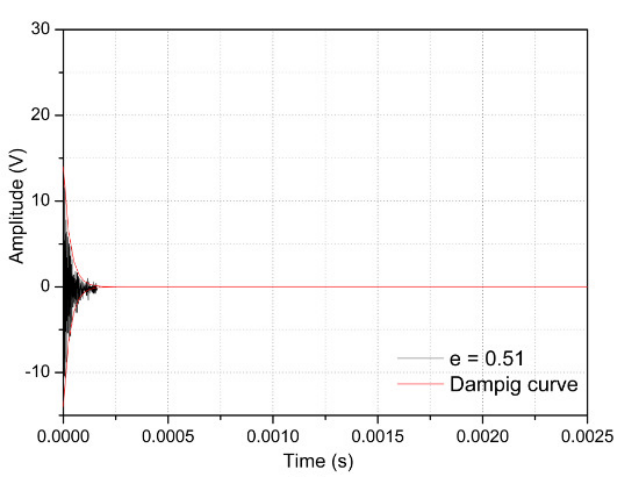

(a)

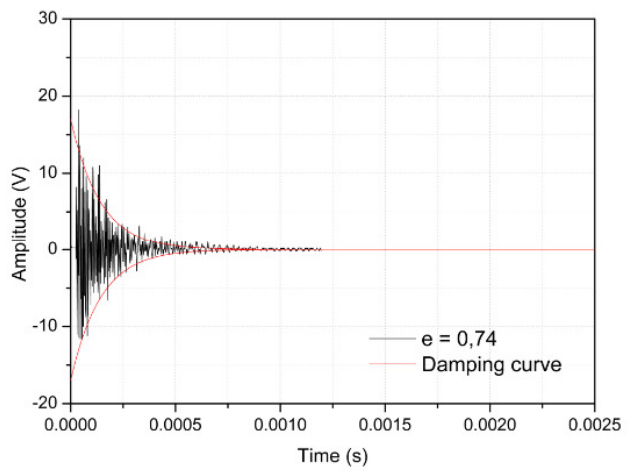

(c)

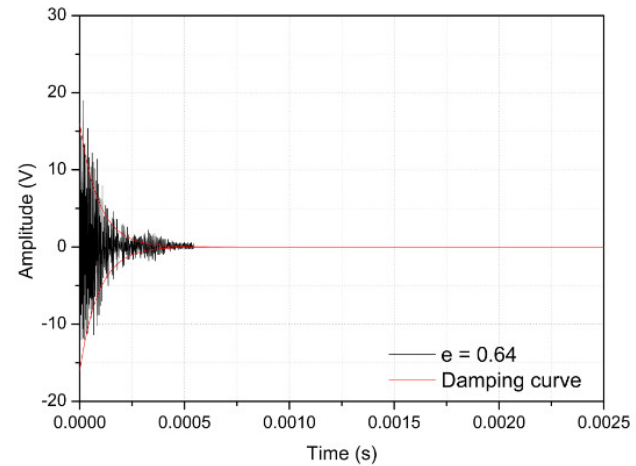

(b)

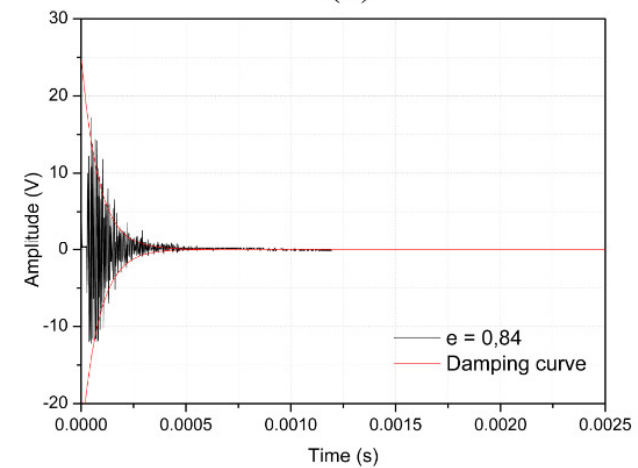

(d)

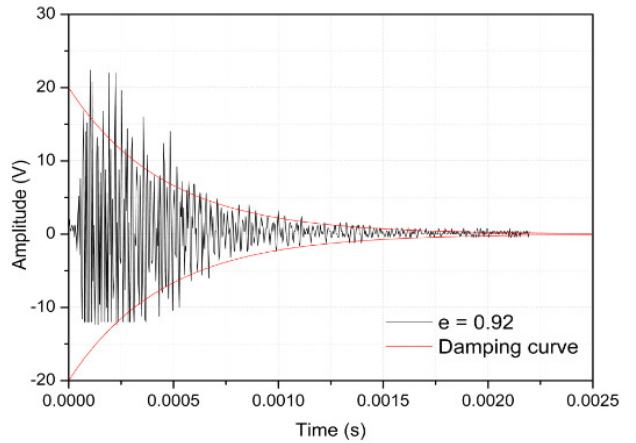

(e)

Figure 1. Waveforms for satured sand of $\mathrm{D}_{50}=0.40 \mathrm{~mm}$. (a) $\mathrm{e}=0.51$; (b) $\mathrm{e}=0.64 ;$ (c) $\mathrm{e}=0.74$; (d) $\mathrm{e}=0.84$; (e) $\mathrm{e}=0.92$ 
Similar results of wave scattering related to concrete materials were obtained by Quiviger, Payan, Chaix, Garnier and Salin (2012) showing that waveforms presenting widely peaks and valleys, random sizes and with reduced wave energy propose diffuse effects causing by wave's scattering.

Taking the average variation of each ultrasonic pulse characteristic as damping coefficient, amplitude, frequency and UPV of each single sample, it was possible to estimate the void ratio range regarding to these characteristics, as shown in Table 1. Thus, in a statistically way, representative values of each void ratio range, shown in Table 2, were defined enabling a better analysis on the results of each type of sand and comparison between the studied sands.

Table 1. Damping coefficient average variations and voids ratio for sands of $\mathrm{D}_{50}=1.15 \mathrm{~mm}$

\begin{tabular}{llll}
\hline Void ratio range & Variation in voids ratio (\%) & Mean coefficient damping variation (\%) & Average coef. damping \\
\hline $0.53-0.58$ & $7.76 \%$ & $8.63 \%$ & 1.3304182 \\
$0.60-0.65$ & $5.47 \%$ & $2.07 \%$ & 1.4347825 \\
$0.73-0.78$ & $5.24 \%$ & $7.88 \%$ & 1.5764968 \\
$0.83-0.87$ & $3.92 \%$ & $3.03 \%$ & 1.6346784 \\
$0.91-1.11$ & $18.02 \%$ & $7.95 \%$ & 1.6762169 \\
\hline
\end{tabular}

Table 2. Values of void ratio and damping coefficient average, including standard deviation (SD) for sands of D50 $=1.15 \mathrm{~mm}$

\begin{tabular}{lllllll}
\hline \multirow{2}{*}{ Void ratio } & \multicolumn{5}{c}{ Average coef. damping } \\
& $\mathrm{D}_{50}=1.15 \mathrm{~mm}$ & $\mathrm{SD}$ & $\mathrm{D}_{50}=0.4 \mathrm{~mm}$ & $\mathrm{SD}$ & $\mathrm{D}_{50}=1.0 \mathrm{~mm}$ & $\mathrm{SD}$ \\
\hline 0.55 & 0.01330 & 0.00052 & 0.00600 & 0.00028 & 0.00754 & 0.00054 \\
0.65 & 0.01435 & 0.00018 & 0.00756 & 0.00027 & 0.00963 & 0.00028 \\
0.75 & 0.01576 & 0.00044 & 0.01002 & 0.00001 & 0.01210 & 0.00076 \\
0.85 & 0.01635 & 0.00027 & 0.01116 & 0.00058 & 0.01020 & 0.00092 \\
0.95 & 0.01601 & 0.00063 & 0.00990 & 0.00032 & 0.00889 & 0.00008 \\
\hline
\end{tabular}

Samples evaluated in this paper, showed a good correlation between the average of damping coefficient and the void ratio, as illustrated in Figure 2. Can be seen that low voids ratio, show a lower damping coefficient. The addition of water in the medium is detected by ultrasonic wave through its attenuation, increasing the damping coefficient.

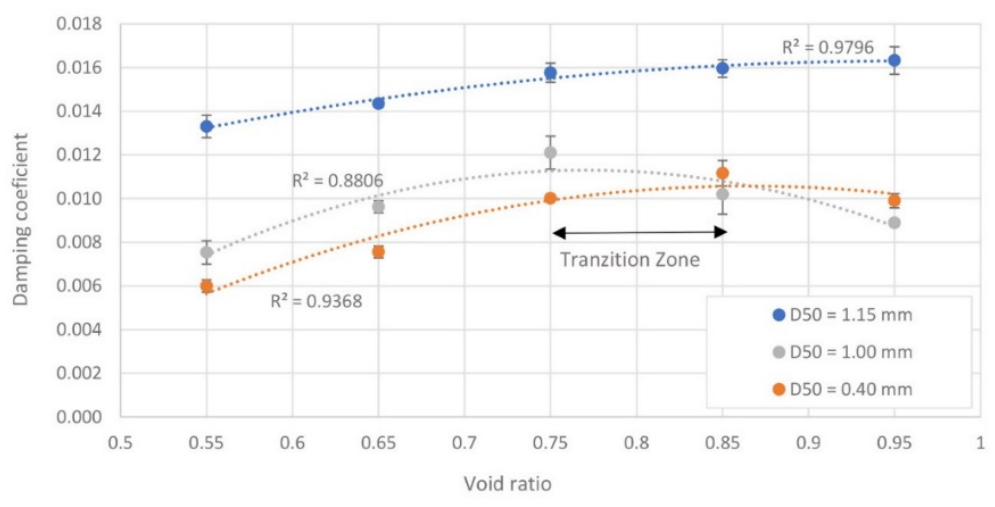

Figure 2. Relationship between void ratio and damping coefficient.

Sands of $D_{50}=0.4 \mathrm{~mm}$ and $D_{50}=1.0 \mathrm{~mm}$, with voids ratio starting from 0.75 show a reduction on damping coefficient. Sands with $\mathrm{D}_{50}=1.15 \mathrm{~mm}$ suggest a reduction on damping coefficient starting from void ratio above 0.85 . Thus, a change in dominant medium (sand $\mathrm{X}$ water) in the soil matrix can be identified, and water becomes predominat. For voids ratio above 0.75 , a smaller variation on damping coefficient average is observed, indicating that water addition does not influence on significant changes in the structure of the soil's matrix and its behavior. 
At this point, it is possible to observe on Figure 2, the larger the median particle size, the greater the damping coefficient. Sands of $\mathrm{D}_{50}=1.15 \mathrm{~mm}$ which have more uniform grains, obtained the highest damping coefficients with a significant difference, approximately $70 \%$ of this sand compared to the others type.

\subsection{Amplitude}

The maximum amplitude average for three types of sand is shown in Table 3 and a correlation with the void ratio is presented in Figure 3 According to Gheibi and Hedayat (2018) the maximum amplitude average decreases with the increase of void ratio.

Table 3 . The maximum amplitude averages and voids ratio

\begin{tabular}{lllllll}
\hline \multirow{2}{*}{ Void ratio } & \multicolumn{5}{c}{ Average Maximum Amplitude (V) } \\
& $\mathrm{D}_{50}=1.15 \mathrm{~mm}$ & $\mathrm{SD}$ & $\mathrm{D}_{50}=0.40 \mathrm{~mm}$ & $\mathrm{SD}$ & $\mathrm{D}_{50}=1.00 \mathrm{~mm}$ & $\mathrm{SD}$ \\
\hline 0.55 & 15.30 & 0.80 & 18.88 & 1.10 & 16.50 & 1.10 \\
0.65 & 12.80 & 0.20 & 18.07 & 0.50 & 14.50 & 0.15 \\
0.75 & 7.80 & 0.15 & 17.05 & 0.00 & 12.35 & 0.30 \\
0.85 & 12.10 & 0.40 & 20.13 & 0.50 & 19.15 & 0.25 \\
0.95 & 13.00 & 0.50 & 20.93 & 1.40 & 18.05 & 0.85 \\
Water & 26.6 & & & & & \\
\hline
\end{tabular}

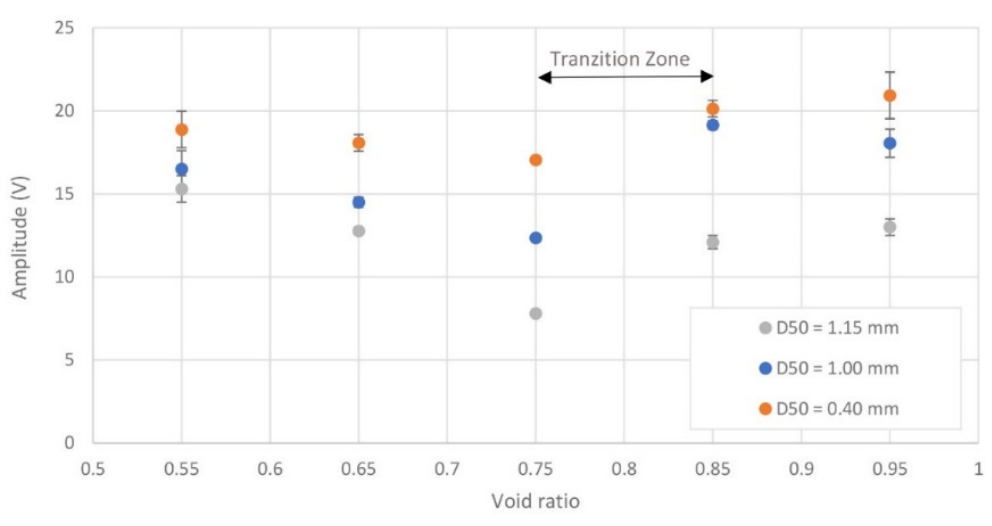

Figure 3. Relationship between void ratio and amplitude for sandy soils.

As shown in Figure 3, similar results were obtained for samples with voids ratio up to 0.75 , describing that the maximum amplitude decreases while voids ratio increases. For samples with voids ratio greater than 0.75 , there are an increase in maximum amplitude average. This change amplitude behavior indicates a significant transformation in soil's matrix, suggesting an existence of transition zones among materials. Gheibi and Hedayat (2018) mention that changes in the maximum amplitude response in granular materials are dependent on the contact area and pore size between particles. Therefore, for samples with void ratio greater than 0.75 , water has a strong influence on the medium, increasing amplitude.

It was also noticed in Figure 3 that samples with a smaller median particle size $\left(\mathrm{D}_{50}\right)$ have a greater maximum amplitude. These results corroborate the results from Gheibi and Hedayat (2018) and Dutta et al. (2019).

Figure 4 presents a statistics correlation between relative denstity $\left(D_{r}\right)$, damping coefficient and amplitude for the studied sands. Chian, Tan and Sarma (2017) described that, when $\mathrm{D}_{\mathrm{r}}=100 \%$ implies that the sand grains are arranged such that the volume of voids within the grains is the minimum and a $D_{r}=0 \%$ means that the sand grains are arranged such that the total volume of voids is the maximum. Thus, as observed on Figure 4 there is a influence of grain size and relative density over ultrasonic propreties also, it is possible to note same behavior of utrasonic propreties: increasing maximum amplitude and decreasing damping coefficiente, means increasing $D_{r}$. Figure $4(c)$ shows a small maximum amplitude variation and huge damping coefficiente values due to the influency of uniform grains size.

Therefore, in addition to a good correlation existence between voids ratio, damping coefficient and amplitude, it is possible to identify transition zones at the same void ratio range. 

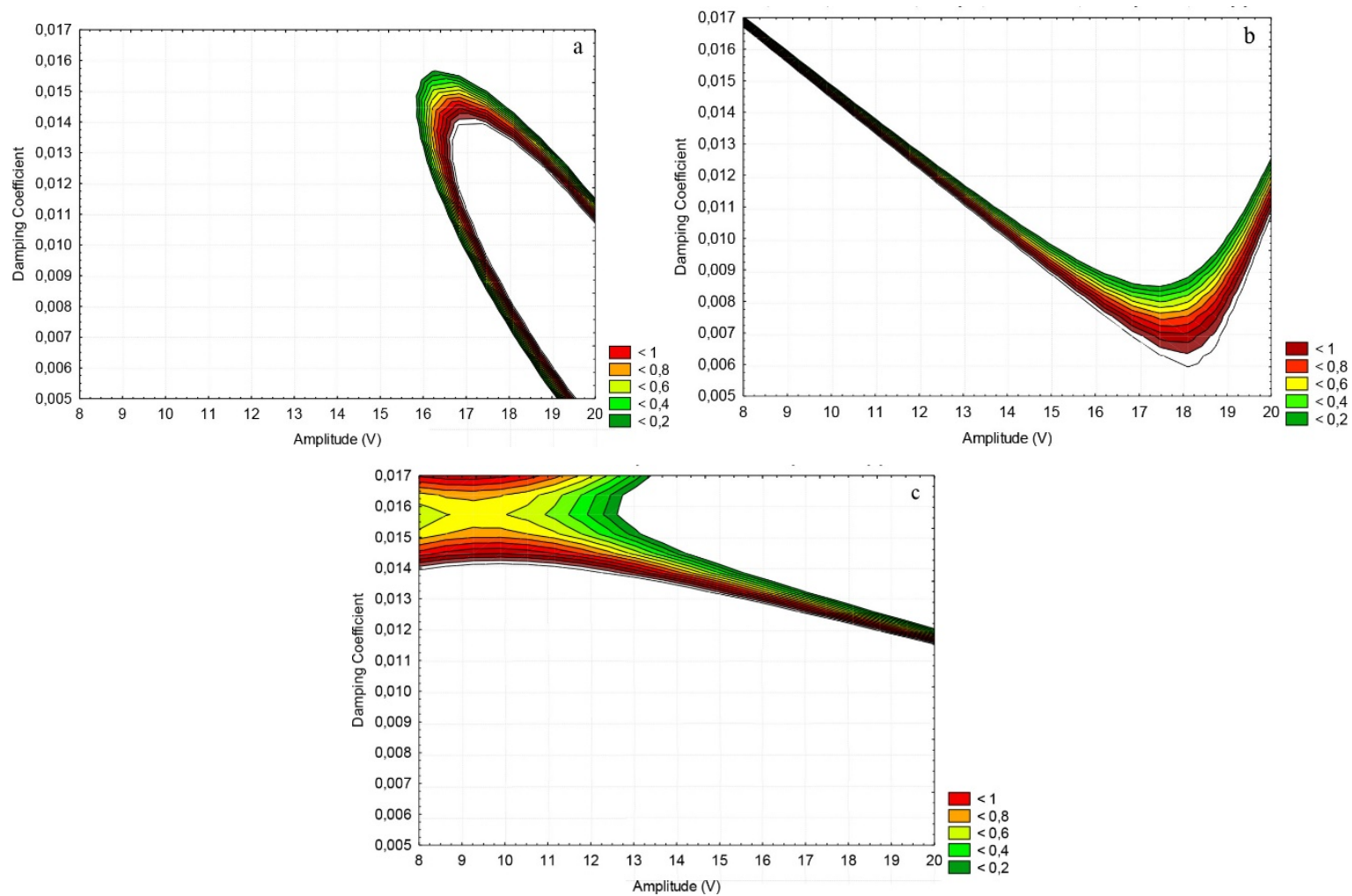

Figure 4. Statistics correlation with relative denstity $\left(D_{r}\right)$, Damping coefficient and amplitude for (a) $D_{50}=0.4$ $\mathrm{mm},(\mathrm{b}) \mathrm{D}_{50}=1.00 \mathrm{~mm}$ and (c) $\mathrm{D}_{50}=1.15 \mathrm{~mm}$.

\subsection{Dominant Frequency}

Figure 5 shows the variation of maximum dominant frequency by each void ratio range.

It is observed that in particle's domain area the dominant frequency decreases while $\mathrm{D}_{50}$ increases. The same result was not found after the transition zone, which indicates the water predominance in the medium and, thereby, the same dominant frequency, regardless of the grain size. Dutta et al. (2019) demonstrate that the variation in the ratio of fast Fourrier transforms through received signal and the transmitted signal, for different values of $\mathrm{D}_{50}$ at a given frequency, decreases while $\mathrm{D}_{50}$ increases.

Finally, through the analysis of maximum amplitude, damping coefficient and dominant frequency, it was possible to identify voids range and also transition zones through characteristics of ultrasonic waves regarding to granular sandy soils.

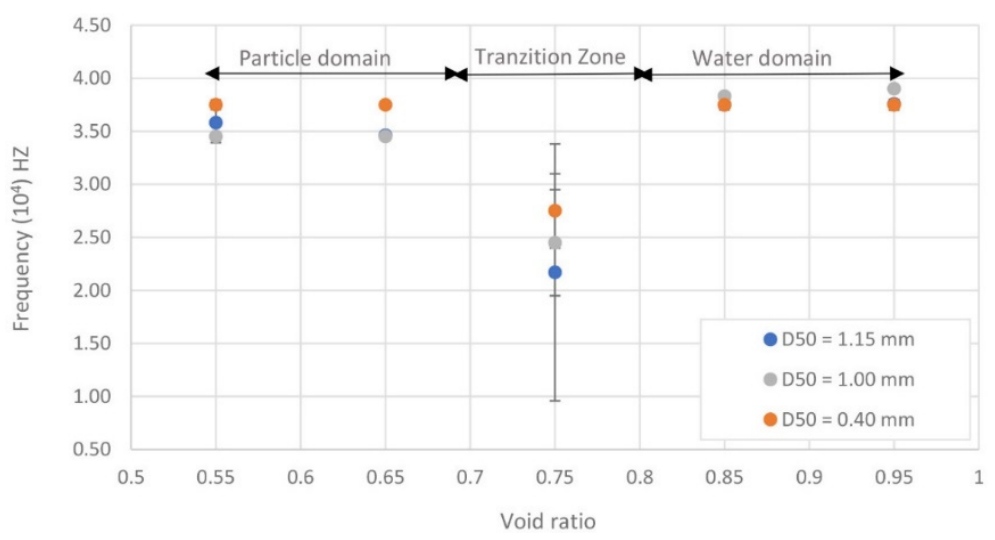

Figure 5. Variation between the dominant frequency average and voids ratio. 


\subsection{Ultrasonic Pulse Velocity}

According to Dutta et al. (2019) S-wave and P-wave are insensitive to median particle size. The results presented in Figure 5 demonstrate a small variation in ultrasonic velocity measurements considering the median particle size.

Zimmer (2004), Xu et al. (2013), Gheibi and Hedayat (2018) and Dutta et al. (2019) show that ultrasonic velocity measurements decrease as increasing the void ratio. Also Figure 6 shown a reduction in velocity while increasing voids ratio up to 0.7 , after this value, there is an increase on ultrasonic pulse velocity. As mentioned by Makse et al. (2004) and Zimmer (2004), ultrasonic velocity measurements depends not only on the solid particle response, but also on the arrangement and inner contacts of soils. Thus, increasing velocity indicates the moment when the water becomes the dominant medium, thereby, the velocity turns into a representative value for the water, equal to $1480 \mathrm{~m} / \mathrm{s}$ according to the literature.

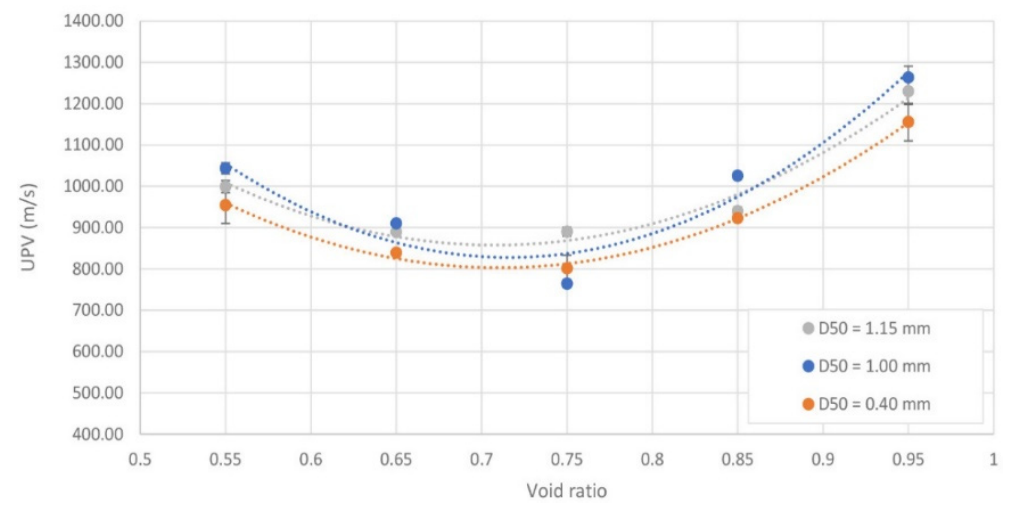

Figure 6. Variation of void ratio and ultrasonic pulse velocity (UPV) average.

\section{Conclusions}

In this contribution, ultrasonic propreties including, maximum amplitude, damping coefficient, dominant frequency, and ultrasonic pulse velocity for saturated sands were analysed and conclude that:

$\mathrm{i}$ - the three types of sands showed damping coefficient average decreases with the reduction of $\mathrm{D}_{50}$.

ii - regardeless to $D_{50}$, damping coefficient average increases as increasing of voids ratio up to 0.75 . After this voids ratio value there is a decreasing on damping coefficient justified by the change of domain of soil's matrix.

iii - the maximum amplitude average decreases with increasing of $\mathrm{D}_{50}$, and also this maximum amplitude average decreases with increasing of voids ratio (up to 0.75 ), meaning that sand is the dominant medium.

iv - the wider variation in frequency for void ratio of 0.75 suggests the existence of a transition zone while lower values of $\mathrm{D}_{50}$ reported higher dominant frequencies.

$\mathrm{v}$ - the ultrasonic velocity measurements decrease with increasing of voids ratio while sand is the dominant medium. The ultrasonic velocity measurements for compression waves are $\mathrm{D}_{50}$ independents.

Therefore, this study newly demonstrated a feasible technique to investigate void ratio of granular saturated soils using direct measurements of the ultrasonic wave characteristics, which is an advancement to the sandy soils characterization.

\section{Acknowledgments}

The authors thank Universidade Federal Fluminense and Coordenação de Aperfeiçoameno de Pessoal de Nível Superior - Brasil (CAPES) for the technical and materials support of this research.

\section{Conflict of interests}

The authors declare that there is no conflict of interests regarding the publication of this paper.

\section{References}

Canivell, J., Martin-del-Rio, J. J., Alejandre, F. J., García-Heras, J., \& Jimenez-Aguilar, A. (2018). Considerations on the physical and mechanical properties of lime-stabilized rammed earth walls and their evaluation by ultrasonic pulse velocity testing. Constr Build Mater, 191, 826-36. https://doi.org/10.1016/j.conbuildmat.2018.09.207 
Chian, S. C., Tan, B. C. V., \& Sarma, A. (2017). Projectile penetration into sand: Relative density of sand and projectile nose shape and mass. International Journal of Impact Engineering, 103, 29-37. https://doi.org/10.1016/j.ijimpeng.2017.01.002

Choobbasti, A. J., Samakoosh, M. A., \& Kutanaei, S. S. (2019). Mechanical properties soil stabilized with nano calcium carbonate and reinforced with carpet waste fibers. Constr. Build Mater, 211, 1094-104. https://doi.org/10.1016/j.conbuildmat.2019.03.306

Das, B. M., \& Sobhan, K. (2014). Fundamentos de Engenharia Geotécnica. Cengage Le. São Paulo.

Dutta, T. T., Otsubo, M., Kuwano, R., \& O’Sullivan, C. (2019). Stress wave velocity in soils: Apparent grain-size effect and optimum input frequencies. Géotechnique Lett, 9, 340-347. https://doi.org/10.1680/jgele.18.00219

Ferronatto, D. N. L. (2016). Determinação do fator de amortecimento de materiais cerâmicos por ultrassom. (dissertation). Universidade Federal Fluminense, Volta Redonda, Brazil.

Gheibi, A., \& Hedayat, A. (2018). Ultrasonic investigation of granular materials subjected to compression and crushing. Ultrasonics, 87, 112-25. https://doi.org/10.1016/j.ultras.2018.02.006

Knuth, M. W., Tobin, H. J., \& Marone, C. (2013). Evolution of ultrasonic velocity and dynamic elastic moduli with shear strain in granular layers. Granul Matter, 15, 499-515. https://doi.org/10.1007/s10035-013-0420-1

Makse, H. A., Gland, N., Johnson, L., \& Schwartz, L. (2004). Granular packings: Nonlinear elasticity, sound propagation, and collective relaxation dynamics. Phys Rev E - Stat Physics, Plasmas, Fluids, Relat Interdiscip Top, 70, 1-19. https://doi.org/10.1103/PhysRevE.70.061302

Nourani, H. M., Taheri Moghadder, M., \& Safari, M. (2017). Classification and assessment of rock mass parameters in Choghart iron mine using P-wave velocity. J Rock Mech Geotech Eng, 9, 318-28. https://doi.org/10.1016/j.jrmge.2016.11.006

Prasad, M., \& Meissner, R. (1992). Attenuation mechanisms in sands: Laboratory versus theoretical (Biot) data. Geophysics, 57, 710-719. https://doi.org/10.1190/1.1443284

Pu, S., Hong, B., Liu, X., Xu, F., \& Shan, H. (2019). Detection Technology of Foamed Mixture Lightweight Soil Embankment Based on Ultrasonic Wave Transmission Method. Adv Mater Sci Eng, 1-15. https://doi.org/10.1155/2019/9654819

Pyrak-Nolt, L., \& Nolte, D. D. (1992). Frequency Dependece of Fracture Stiffness. Geophys R F Seach, 19, 325338. https://doi.org/10.1029/91GL03179

Wiggenhauser, H., Samokrutov, A., Mayer, K., Krause, M., Alekhin, S., \& Elkin, V. (2017). Large aperture ultrasonic system for testing thick concrete structures. $J$ Infrastruct Syst, 23, 1-9. https://doi.org/10.1061/(ASCE)IS.1943-555X.0000314

Xu, X., Cheng, Y., \& Ling, D. (2013). The influence of void ratio on small strain shear modulus of granular materials: A micromechanical perspective. AIP Conf Proc, 1542, 201-214. https://doi.org/10.1063/1.4811902

\section{Copyrights}

Copyright for this article is retained by the author(s), with first publication rights granted to the journal.

This is an open-access article distributed under the terms and conditions of the Creative Commons Attribution license (http://creativecommons.org/licenses/by/4.0/). 